\title{
Numerical method for angle-of-incidence correction factors for diffuse radiation incident photovoltaic modules
}

\author{
Bill Marion *
}

\begin{abstract}
A numerical method is provided for solving the integral equation for the angle-of-incidence (AOI) correction factor for diffuse radiation incident photovoltaic (PV) modules. The types of diffuse radiation considered include sky, circumsolar, horizon, and ground-reflected. The method permits PV module AOI characteristics to be addressed when calculating AOI losses associated with diffuse radiation. Pseudo code is provided to aid users in the implementation, and results are shown for PV modules with tilt angles from $0^{\circ}$ to $90^{\circ}$. Diffuse AOI losses are greatest for small PV module tilt angles. Including AOI losses associated with the diffuse irradiance will improve predictions of PV system performance.
\end{abstract}

Key words: Numerical solution; Angle-of-incidence losses; Diffuse solar radiation; Model.

\section{Introduction}

The angle-of-incidence (AOI) is the angle between the normal to the photovoltaic (PV) module surface and the vector of the incoming radiation. When the AOI is increased, an increased percentage of the in-plane irradiance is reflected from the surface of a PV module. This reduces the in-plane irradiance reaching the PV cells that generates electricity. To account for this reduction when modeling PV performance, the in-plane irradiance is multiplied by an AOI correction factor (from 0 to 1). The AOI correction factor is the in-plane irradiance reaching the PV cells divided by the in-plane irradiance reaching the PV cells when the AOI is normal to the PV module surface. Other terms for the AOI correction factor include: transmittance for diffuse radiation (Brandemuehl and Beckman, 1980), relative transmittance (Sjerps-Koomen et al., 1996), angular factor (Martin and Ruiz, 2001, 2013), incidence angle modifier (De Soto et al., 2006), and relative light transmission into the module (IEC, 2016).

The AOI correction factor may be represented by a function, $F(\mathrm{AOI})$, that provides a relationship with the AOI. The function may be based on indoor or outdoor tests where the short-circuit current output of the PV module or one of its cells is measured over a range of AOIs. Eq. (1) is the Sandia function as formulated by King et al. (2004) as a $5^{\text {th }}$-order polynomial where the $a$ values are the polynomial curve-fit coefficients:

$$
F(\mathrm{AOI})=a_{0}+a_{1} \cdot \mathrm{AOI}+a_{2} \cdot \mathrm{AOI}^{2}+a_{3} \cdot \mathrm{AOI}^{3}+a_{4} \cdot \mathrm{AOI}^{4}+a_{5} \cdot \mathrm{AOI}^{5}
$$

Eq. (2) is the function as formulated by Martin and Ruiz $(2001,2013)$ using exponentials where $a_{r}$ is the angular loss coefficient determined from a best fit of the measured data. This formulation is used in the International Electrochemical Commission (IEC) standard 61853-2 (IEC, 2016):

$$
F(\mathrm{AOI})=\left[1-\exp \left(-\cos (\mathrm{AOI}) / a_{r}\right)\right] /\left[1-\exp \left(-1 / a_{r}\right)\right]
$$

For PV modules with a flat glass surface, the IEC standard 61853-2 also allows an air-glass model, such as developed by Sjerps-Koomen et al. (1996), to be used in place of measurements. The air-glass model resulted from studies that showed that the air-glass interface dominates the transmittance of radiation relative to normal incidence. Snell's law is used to determine the angle of refraction in the glass and Fresnel equations are used to determine the reflection from the glass surface. From Duffie and Beckman (1991), the reflectance at normal incidence, $r_{0}$, is defined by Eq. (3):

$$
r_{0}=[(n-1) /(n+1)]^{2}
$$

where $n$ is the index of refraction of the PV module cover material. (For glass, $n=1.526$ and $r_{0}$ becomes 0.0434 .) For values of AOI other than for normal incidence, the angle of refraction, $\mathrm{AOI}_{r}$, is determined using Eq. (4) and then the reflectance, $r_{A O I}$, is determined using Eq. (5):

$$
\mathrm{AOI}_{r}=\sin ^{-1}(\sin (\mathrm{AOI}) / n),
$$


Both the direct beam and diffuse irradiance may be corrected for the AOI, with the diffuse irradiance classified by its type: 1) sky — radiation from the sky dome, 2) circumsolar-radiation from the region of the sky near the sun, 3) horizon-radiation from the region of the sky near the horizon, and 4) ground-reflected-radiation reflected from the ground. This classification of the diffuse irradiances is consistent with the popular Perez transposition model where the sky-dome irradiance distribution has an isotropic background with enhanced circumsolar and horizon regions (Perez et al., 1990). Other transposition models use similar classifications for diffuse irradiances. Yang (2016) provides a recent summary of available transposition models and their features.

The circumsolar diffuse irradiance is considered to have the same AOI as the beam irradiance; consequently, their values of $F(\mathrm{AOI})$ are the same. Their AOI is calculated based on the sun's position and the PV module's azimuth and tilt from horizontal. For the other classifications of diffuse irradiance, the PV module receives radiation from angles within its $180^{\circ}$ field of view. If the radiation is considered isotropic - meaning that the radiation intensity is the same and independent of direction - then radiation from directions with large AOIs contribute less to the in-plane irradiance because the cosine of the AOI is less. A smaller amount of radiation will also reach the PV cells because the value of $F(\mathrm{AOI})$ is also less. Based on the work of Brandemuehl and Beckman (1980), Eq. (7) integrates the effect of the AOI for all angles within the field of view to provide an overall AOI correction factor for the diffuse radiation, $F_{d}$ :

$$
F_{d}=\frac{\int_{A} F(\mathrm{AOI}) \cdot \cos (A O I) \mathrm{d} \omega}{\int_{A} \cos (A O I) \mathrm{d} \omega},
$$

where $\omega$ is the solid angle of the incident diffuse irradiance and $A$ is the range of $\omega$. The radiation's $F(\mathrm{AOI})$ is weighted by its contribution to the in-plane irradiance.

This work provides a method for numerically integrating Eq. (7) to obtain overall AOI correction factors for the sky, horizon, and ground-reflected diffuse irradiance components. To aid users in the implementation of the method, pseudo code is provided in Section 3, and example results for a PV module are provided in Section 4.

\section{Numerical Method}

By definition, the solid angle subtended by an object is the area projected onto the surface of the unit sphere, radius of one, with the vertex of the solid angle located at the center of the unit sphere. The dimensionless unit of the solid angle is the steradian. About a point, the total solid angle is $4 \pi$, which is the same as the surface area of a sphere with a radius of one.

In Fig. 1, an elemental surface area, $\mathrm{d} A$, of the sky viewed by the PV module is projected onto the surface of the unit sphere. The resulting projected surface area, $\mathrm{d} A_{s}$, equals the solid angle $\mathrm{d} \omega$. To facilitate calculating values of $\mathrm{d} A_{s}$, the $\phi-\psi$ coordinate system shown in Fig. 2 is used to define $\mathrm{d} A_{s}$, where $\phi$ is the angle between the zenith and a line from the center of the sphere to the $\mathrm{d} A_{s}$ and $\psi$ is the angle from north for a line from the center of the sphere to the vertical projection of the $\mathrm{d} A_{s}$ in the horizontal plane. From elementary geometry, the surface area of $\mathrm{d} A_{s}$ may be calculated using Eq. (8):

$$
\mathrm{d} A_{s}=\left(\psi_{2}-\psi_{1}\right) \cdot\left[\cos \left(\phi_{1}\right)-\cos \left(\phi_{2}\right)\right]
$$

where $\psi$ is in radians and the subscripts define the range of angles, with a subscript of one corresponding to the minimum angle and a subscript of two corresponding to the maximum angle. Using the average values of $\phi$ and $\psi$ to represent the center of the $\mathrm{d} A_{s}$ and recognizing that the coordinate system is the same as that used for the sun's position in the sky, an equation from Iqbal (1983) provides the AOI:

$$
\mathrm{AOI}=\cos ^{-1}\left[\cos (\beta) \cdot \cos \left(\left(\phi_{1}+\phi_{2}\right) / 2\right)+\sin (\beta) \cdot \sin \left(\left(\phi_{1}+\phi_{2}\right) / 2\right) \cdot \cos \left(\left(\psi_{1}+\psi_{2}\right) / 2-\gamma\right)\right],
$$

where $\beta$ is the PV module tilt angle from horizontal and $\gamma$ is the PV module azimuth angle from north. 


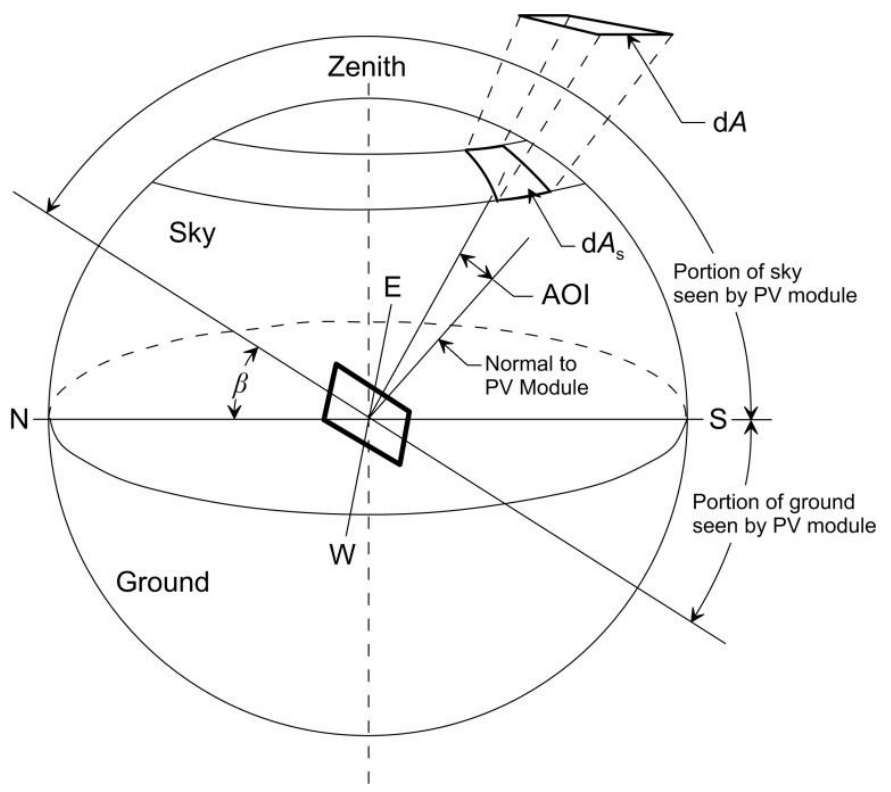

Fig. 1. PV module located at the center of the unit sphere showing the regions of the sky and ground within the PV module's field of view, the elemental area, $\mathrm{d} A$, projected onto the unit sphere, and the resulting angle of incidence, AOI, between the projected elemental area, $\mathrm{d} A_{s}$ and the normal to the PV module.

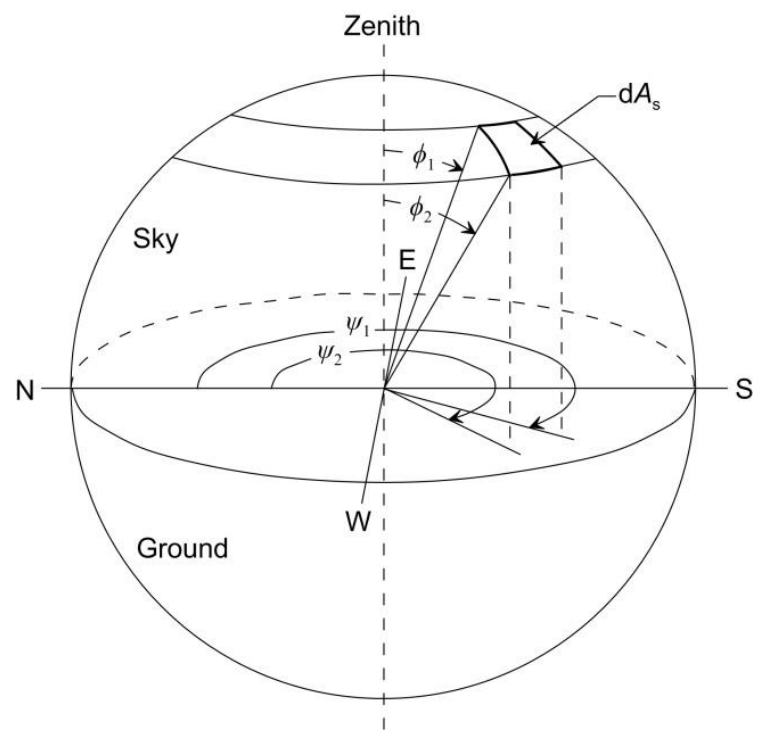

Fig. 2. The $\phi-\psi$ coordinate system for defining the location of the elemental area, $\mathrm{d} A_{s}$, on the unit sphere.

Eq. (10) is used for the numerical method and is essentially equivalent to Eq. (7), with $\mathrm{d} \omega$ replaced by $\mathrm{d} A_{s}$ and the integrals replaced by summations over the appropriate ranges of $\phi$ and $\psi$ that are within the field-of-view of the PV module $(\mathrm{AOI}<\pi / 2)$ and for when $\phi$ is valid for the type of diffuse irradiance considered, per Table 1:

$$
F_{d}=\frac{\sum_{\phi=0}^{\phi=\pi} \sum_{\psi=0}^{\psi=2 \pi} F(\mathrm{AOI}) \cdot \cos (\mathrm{AOI}) \cdot \mathrm{d} A_{s}}{\sum_{\phi=0}^{\phi=\pi} \sum_{\psi=0}^{\psi=2 \pi} \cos (\mathrm{AOI}) \cdot \mathrm{d} A_{s}} \quad[\mathrm{AOI}<\pi / 2 ; \phi \text { from Table } 1]
$$

Table 1 lists the valid ranges for AOI and $\phi$ when implementing Eq. (10) for the different types of diffuse irradiances. Although the horizon diffuse irradiance is considered to be from the horizon $(\phi=\pi / 2)$, a half degree above the horizon is included to provide sufficient data for the numerical method $(89.5 \cdot \pi / 180 \leq \phi \leq \pi / 2)$. 


\begin{tabular}{lrr} 
Table 1 & \\
Valid ranges of AOI and $\phi$ for performing summations in Eq. (10) \\
\hline Diffuse Irradiance & AOI & $\phi$ \\
\hline Sky & $<\pi / 2$ & $\leq \pi / 2$ \\
Horizon & $<\pi / 2$ & $\geq 89.5 \cdot \pi / 180$ and $\leq \pi / 2$ \\
Ground-reflected & $<\pi / 2$ & $\geq \pi / 2$ \\
\hline
\end{tabular}

As a check on the implementation of Eq. (10), it is useful to recognize that the denominator divided by $\pi$ is the numerical approximation of the configuration factor, $C F$. The $C F$ is the fraction of energy leaving a surface that is received by another surface. For our application, the energy leaving a surface is the sky, horizon, or ground-reflected diffuse irradiance. The receiving surface is the PV module. The mathematical solution for the $C F$ is $(1+\cos \beta) / 2$ for the sky irradiance and $(1-\cos \beta) / 2$ for ground-reflected irradiance (Iqbal, 1983). Comparing the denominator of Eq. (10) divided by $\pi$ with the mathematical solution checks the implementation of the method. (The denominator is divided by $\pi$ because the unit sphere sky dome projected onto the horizontal is equal to $\pi$.)

\section{Pseudo Code}

The following pseudo code determines the result of Eq. (10) and may be implemented in various programming languages.

INPUT $\quad \beta$ and type of diffuse (sky, horizon, or ground-reflected)

OUTPUT $\quad F_{d}$ (diffuse AOI correction factor)

Step 1 Set Numerator Sum and Denominator Sum to zero

Set $\gamma$ to $\pi$ (or any value between 0 and $2 \pi$ )

Set $N$ to the number of increments of $\phi$ from 0 to $\pi$

( $N=180$ for $1^{\circ}$ increments, $N=1800$ for $0.1^{\circ}$ increments)

Set $a i=\pi / N$ (the angular increment factor, radians)

Set $i=0$ (integer counter for incrementing $\phi$ )

Step 2 While $i<N$, do Steps 3-12 (for $\phi$ from 0 to $\pi$ )

Step $3 \quad \phi_{1}=i \cdot a i \quad\left(\right.$ assign $\phi_{1}$ and $\left.\phi_{2}\right)$

$\phi_{2}=\phi_{1}+a i$

Step 4 Set $j=0$ (integer counter for incrementing $\psi$ )

Step 5 While $j<2 N$, do Steps 6-11 (for $\psi$ from 0 to $2 \pi$ )

Step $6 \quad \psi_{1}=j \cdot a i \quad\left(\right.$ assign $\psi_{1}$ and $\psi_{2}$ )

$\psi_{2}=\psi_{1}+a i$

Step 7 Calculate $\mathrm{d} A_{s}$ with Eq. (8)

Calculate AOI with Eq. (9)

Step 8 If AOI, $\phi_{1}$, and $\phi_{2}$ valid per Table 1 , do Steps 9-10

Step 9 Calculate $F(\mathrm{AOI}$ ) with Eq. (1), (2), or (3) through (6)

Step 10 Add $F(\mathrm{AOI}) \cdot \cos (\mathrm{AOI}) \cdot \mathrm{d} A_{s}$ to Numerator Sum

Add $\cos (\mathrm{AOI}) \cdot \mathrm{d} A_{s}$ to Denominator Sum

Step $11 \operatorname{Set} j=j+1$

Step 12 Set $i=i+1$

Step $13 F_{d}=$ Numerator Sum / Denominator Sum, with Eq. (10)

Step 14 OUTPUT $\left(F_{d}\right)$

\section{Results and Discussion}

The pseudo code from Section 3 was implemented to determine the diffuse AOI correction factors for PV modules with two different types of front surfaces: glass $(n=1.526)$, and antireflection (AR) coated glass $(n=1.3)$. The air-glass model, Eqs. (3) through (6), was used for the $F(\mathrm{AOI})$ and is also shown in Fig. 3 as the correction factor, $F_{b}$, for the beam and circumsolar irradiance. A value of $\pi$ was used for $\gamma$, but any value between 0 and $2 \pi$ provides the same result because the radiation intensity of the sky, horizon, and ground-reflected radiation is considered isotropic and therefore not dependent on $\gamma$. The solution is also valid for both the north and south hemispheres.

To show the dependency on $\beta$, the correction factor for the diffuse sky irradiance, $F_{s k y}$, the correction factor for the diffuse horizon irradiance, $F_{h o r}$, and the correction factor for the diffuse ground-reflected irradiance, $F_{\text {grd }}$, were determined for values of $\beta$ 
from $0^{\circ}$ to $90^{\circ}$ at $1^{\circ}$ intervals. Fig. 4 illustrates the results for $F_{s k y}$ and Fig. 5 illustrates the results for both $F_{g r d}$ and $F_{h o r}$. For $F_{s k y}$ and $F_{g r d}$, a value of $N$ equal to 180 was used to set $1^{\circ}$ angular increments for $\phi$ and $\psi$. This permitted the program execution to be completed within a few seconds and the denominator of Eq. (10) divided by $\pi$ to be accurate to four decimal places (when compared to the mathematical solution of $(1+\cos \beta) / 2$ for the diffuse sky irradiance or $(1-\cos \beta) / 2)$ for the diffuse groundreflected irradiance). For $F_{h o r}$, which is evaluated for a $0.5^{\circ}$ band at the horizon, a value of $N$ equal to 1,800 was used for angular increments of $0.1^{\circ}$. As expected, the AR-coated glass exhibits better AOI characteristics.

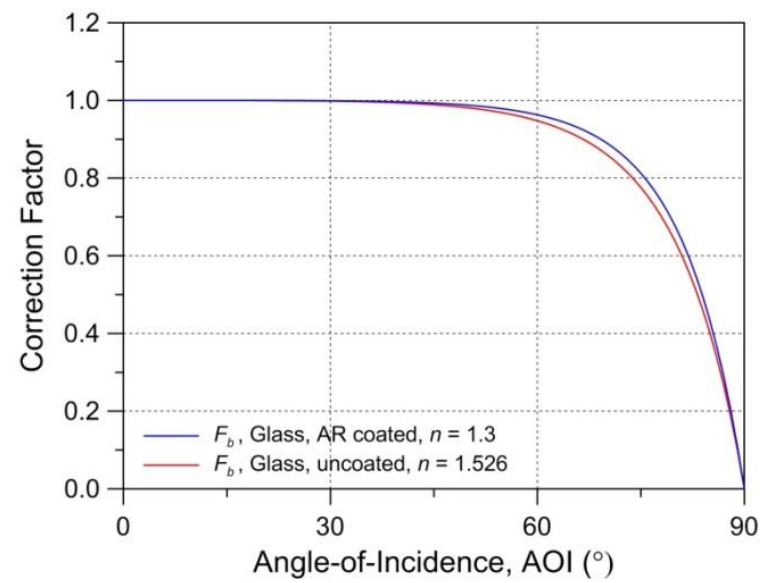

Fig. 3. Angle-of-incidence (AOI) correction factor for beam and circumsolar irradiance, $F_{b}$, for two types of PV module front-cover materials: uncoated glass with a refractive index, $n$, of 1.526 and antireflection (AR) coated glass with $n=1.3$.

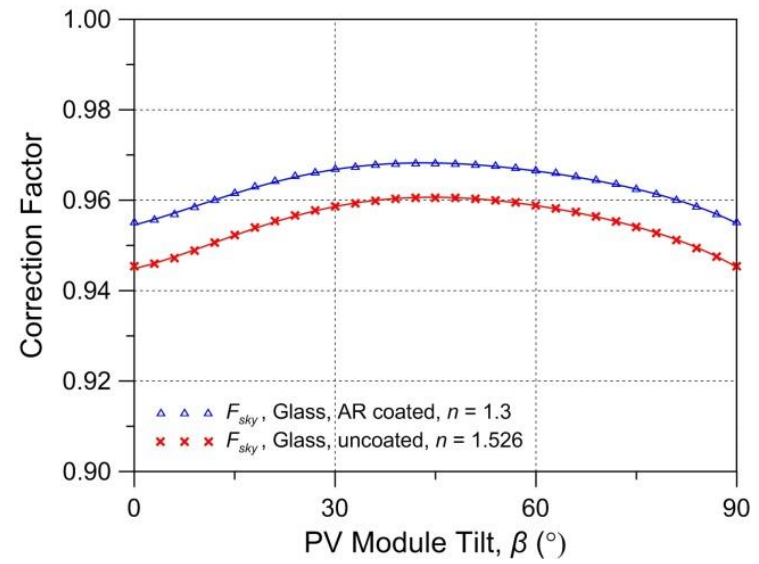

Fig. 4. Angle-of-incidence (AOI) correction factor as a function of the PV module tilt angle from horizontal, $\beta$, for the diffuse sky irradiance, $F_{\text {sky }}$, for two types of PV module front-cover materials: uncoated glass with a refractive index, $n$, of 1.526 and antireflection (AR) coated glass with $n=1.3$.

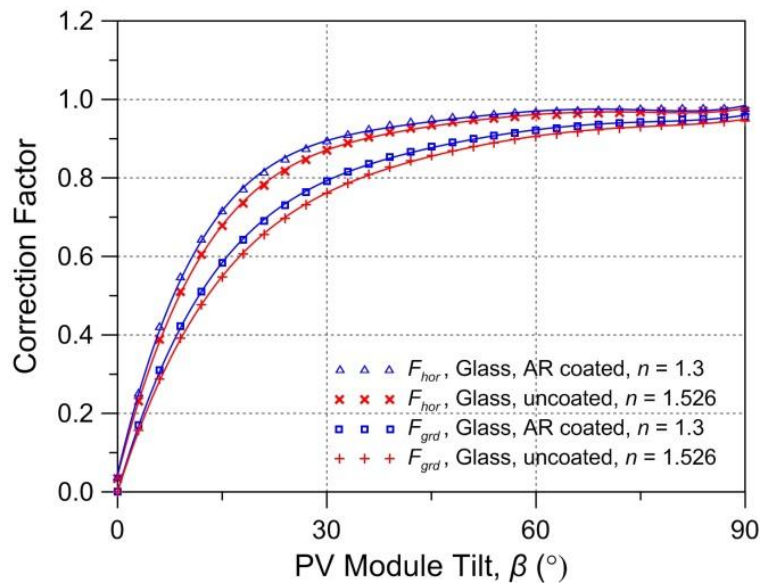


Fig. 5. Angle-of-incidence (AOI) correction factors as a function of the PV module tilt angle from horizontal, $\beta$, for the diffuse horizon, $F_{\text {hor }}$, and ground-reflected irradiance, $F_{g r d}$, for two types of PV module front-cover materials: uncoated glass with a refractive index, $n$, of 1.526 and antireflection (AR) coated glass with $n=1.3$.

As shown in Fig. $4, F_{s k y}$ has a small dependency on $\beta$. This contrasts with $F_{h o r}$ and $F_{g r d}$ in Fig. 5 which shows a large dependency on $\beta$ and approaches zero as $\beta$ approaches zero. In Figs. 4 and 5, the symbols represent the resulting data from the numerical solutions performed at values of $\beta$, and the solid lines are polynomial curve fits of these data. To more readily determine a value of $F_{s k y,}, F_{h o r}$, or $F_{\text {grd }}$ for a value of $\beta$, the polynomial curves represented by Eq. (11) may be used as a substitute for performing a numerical solution:

$$
F_{d}=a_{0}+a_{1} \cdot \beta+a_{2} \cdot \beta^{2}+a_{3} \cdot \beta^{3}+a_{4} \cdot \beta^{4}+a_{5} \cdot \beta^{5},
$$

where values of $a_{\mathrm{n}}$ are the polynomial coefficients and values of $\beta$ are in degrees from 0 to 90 . For the two types of PV module front-cover materials considered, Table 2 provides the polynomial coefficients for $F_{s k y}, F_{h o r}$, and $F_{g r d}$. Unlike $F_{b}$, values of $F_{s k y}$, $F_{h o r}$, and $F_{g r d}$ only need to be determined once when modeling the performance of PV modules with a fixed tilt.

Table 2

Polynomial coefficients for the correction factors $F_{s k v}, F_{h o r}$, and $F_{g r d}$ for two types of PV module front-cover materials.

\begin{tabular}{|c|c|c|c|c|c|c|c|}
\hline \multirow{2}{*}{$\begin{array}{l}\text { PV Module } \\
\text { Front Cover }\end{array}$} & \multirow{2}{*}{$\begin{array}{l}\text { Correction } \\
\text { Factor }\end{array}$} & \multicolumn{6}{|c|}{ Polynomial Coefficients } \\
\hline & & $a_{0}$ & $a_{1}$ & $a_{2}$ & $a_{3}$ & $a_{4}$ & $a_{5}$ \\
\hline \multirow{3}{*}{$\begin{array}{l}\text { Uncoated glass, } \\
n=1.526\end{array}$} & $F_{s k y}$ & $9.4487 \mathrm{E}-01$ & $3.4581 \mathrm{E}-04$ & $1.8524 \mathrm{E}-05$ & $-7.0766 \mathrm{E}-07$ & $8.1577 \mathrm{E}-09$ & $-3.3904 \mathrm{E}-11$ \\
\hline & $F_{\text {hor }}$ & 4.1215E-02 & $6.9495 \mathrm{E}-02$ & $-2.3414 \mathrm{E}-03$ & 4.1474E-05 & $-3.6908 \mathrm{E}-07$ & $1.2917 \mathrm{E}-09$ \\
\hline & $F_{g r d}$ & $6.9258 \mathrm{E}-04$ & $5.5804 \mathrm{E}-02$ & $-1.6406 \mathrm{E}-03$ & $2.7175 \mathrm{E}-05$ & $-2.3399 \mathrm{E}-07$ & $8.0562 \mathrm{E}-10$ \\
\hline \multirow{3}{*}{$\begin{array}{l}\text { AR-coated glass, } \\
n=1.3\end{array}$} & $F_{s k y}$ & $9.5453 \mathrm{E}-01$ & $3.8205 \mathrm{E}-04$ & $1.2345 \mathrm{E}-05$ & $-5.5902 \mathrm{E}-07$ & $6.7806 \mathrm{E}-09$ & $-2.9021 \mathrm{E}-11$ \\
\hline & $F_{\text {hor }}$ & $4.6333 \mathrm{E}-02$ & $7.5181 \mathrm{E}-02$ & $-2.6741 \mathrm{E}-03$ & 4.8924E-05 & $-4.4356 \mathrm{E}-07$ & $1.5696 \mathrm{E}-09$ \\
\hline & $F_{g r d}$ & $1.1497 \mathrm{E}-03$ & $6.0806 \mathrm{E}-02$ & $-1.8826 \mathrm{E}-03$ & $3.2026 \mathrm{E}-05$ & $-2.7921 \mathrm{E}-07$ & $9.6664 \mathrm{E}-10$ \\
\hline
\end{tabular}

\section{Summary}

A numerical method was developed and the pseudo code was presented for solving the integral equation for the AOI correction factor for diffuse radiation incident PV modules. The types of diffuse radiation considered included sky, circumsolar, horizon, and ground-reflected. The method allows the use of an $F(\mathrm{AOI})$ determined with the air-glass model, the Sandia method, or the IEC 61853-2 standard. Results when using the air-glass model were shown for PV modules with $\beta$ from $0^{\circ}$ to $90^{\circ}$, and for both uncoated and AR-coated glass. Diffuse AOI losses were greatest for small PV module tilt angles and for the uncoated glass. To more readily determine a value of $F_{s k y}, F_{h o r}$, or $F_{g r d}$ for a value of $\beta$, polynomial curves were established for use as a substitute for performing a numerical solution.

\section{Acknowledgement}

This work was supported by the U.S. Department of Energy (DOE) under Contract No. DE-AC36-08-GO28308 with the National Renewable Energy Laboratory (NREL). Funding provided by U.S. DOE Office of Energy Efficiency and Renewable Energy Solar Energy Technologies Program.

The U.S. Government retains and the publisher, by accepting the article for publication, acknowledges that the U.S. Government retains a nonexclusive, paid-up, irrevocable, worldwide license to publish or reproduce the published form of this work, or allow others to do so, for U.S. Government purposes.

\section{References}

Brandemuehl, M., Beckman, W., 1980. Transmission of diffuse radiation through CPC and flat plate collector glazings, Solar Energy 24, 511-513.

De Soto, W., Klein, S., Beckman, W., 2006. Improvement and validation of a model for photovoltaic array performance, Solar Energy 80, 76-88.

Duffie, J., Beckman, W., 1991. Solar Engineering of Thermal Processes, $2^{\text {nd }}$ ed. John Wiley \& Sons, Inc., New York.

IEC, 2016. Photovoltaic (PV) module performance testing and energy rating - Part 2: Spectral responsivity, incidence angle and module operating temperature measurements. IEC Standard 61853-2, Geneva, Switzerland. 
Iqbal, M., 1983. An Introduction to Solar Radiation, Academic Press, Inc., New York.

King, D., Boyson, W., Kratochvil, J., 2004. Photovoltaic Array Performance Model, Sandia National Laboratories, Albuquerque, NM.

Martin, N., Ruiz, J., 2001. Calculation of the PV modules angular losses under field conditions by means of an analytical model, Solar Energy Materials \& Solar Cells 70, 25-38.

Martin, N., Ruiz, J., 2013. Corrigendum to calculation of the PV modules angular losses under field conditions by means of an analytical model, Solar Energy Materials \& Solar Cells 110, 154.

Perez, R., Ineichen, P., Seals, R., Michalsky, J., 1990. Modeling daylight availability and irradiance components from direct and global irradiance, Solar Energy 44, 271-289.

Sjerps-Koomen, E.A., Alsema, E.A., Turkenburg, W.C., 1996. A simple model for PV module reflection losses under field conditions, Solar Energy 57, 421-432.

Yang, D., 2016. Solar radiation on inclined surfaces: Corrections and benchmarks, Solar Energy 136: $288-302$. 